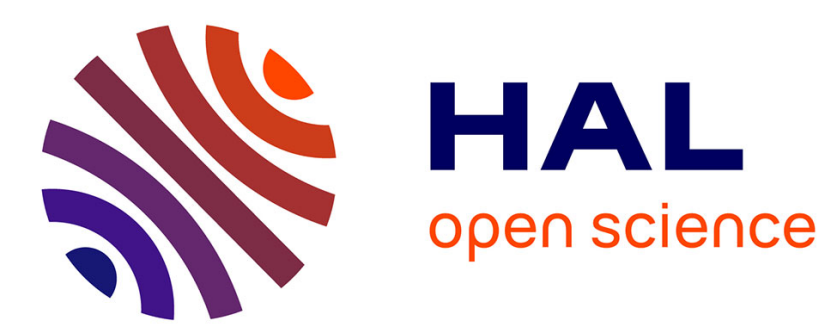

\title{
Wave separation in non-uniform Hopkinson bars using redundant measurements \\ Ramzi Othman
}

\section{To cite this version:}

Ramzi Othman. Wave separation in non-uniform Hopkinson bars using redundant measurements. Journal de Physique IV Proceedings, 2006, 134, pp.571 - 576. 10.1051/jp4:2006134088 . hal01885588

\section{HAL Id: hal-01885588 \\ https://hal.science/hal-01885588}

Submitted on 2 Oct 2018

HAL is a multi-disciplinary open access archive for the deposit and dissemination of scientific research documents, whether they are published or not. The documents may come from teaching and research institutions in France or abroad, or from public or private research centers.
L'archive ouverte pluridisciplinaire HAL, est destinée au dépôt et à la diffusion de documents scientifiques de niveau recherche, publiés ou non, émanant des établissements d'enseignement et de recherche français ou étrangers, des laboratoires publics ou privés. 


\title{
Wave separation in non-uniform Hopkinson bars using redundant measurements
}

\author{
R. Othman ${ }^{1}$ \\ ${ }^{1}$ Institut de Recherche en Génie Civil, École Centrale de Nantes, 1 rue de la Noë, \\ BP. 92101, 44321 Nantes Cedex 3, France
}

\begin{abstract}
In this paper we generalize the BCGO-wave separation method (Bussac, 2002, J. Phys. Mech. Solids, 50, 321-349) based on the maximum of likelihood principle to non-uniform rods. To achieve this aim, the force and the particle velocity at any cross-section are expressed as functions of the force and the particle velocity at the origin instead of the expressing them as function of the forward and downward waves. Due to this generalisation, one can perform long duration and medium strain-rate (1-100/s) tests using Hopkinson bars not only in the case of compression loadings but also for example dynamic bending and high-temperature tests. The new method is checked numerically on a stepped elastic bar. It shows good behaviour against noise.
\end{abstract}

\section{INTRODUCTION}

The Split Hopkinson bar apparatus is an unavoidable test in the dynamic characterization of materials. This is due to the simple and accurate modelling of wave propagation in long elastic and viscoelastic bars. For long time, this system is limited to the high strain-rate loading (approximately from 100 to $5000 / \mathrm{s}$ ). The restriction origin is the wave separation technique which induces maximum test duration. Many authors, mainly in the last decade, proposed alternative wave separation techniques with no limitation on the test duration so to enlarge Hopkinson bar technique to the medium strain-rate loadings [2-4, 6-9]. The most powerful method is the one proposed by Bussac et al. [2]. It is based on the analysis of redundant measurements using the maximum of likelihood method which has the advantage to take into account noise. All these techniques are based on the assumption that the involved bars are uniforms. This is the case, when compressive load are applied. For other kinds of loading (for example tension or bending), a device is needed between the bars and the tested sample. This device induces parasite reflections of the wave in the bars due to mechanical impedance variation. Besides, when dynamic tests are carried out at temperatures different of that of the room, it is necessary to cool or to heat so to maintain the sample at the desired temperature. The bar sides are inescapably affected. An impedance gradient takes place at the bar ends. Lundberg et al. [4] and Bacon \& Brun [1] proposed wave separation solutions taking into account the bar non-uniformity using a transfer matrix approach. In this paper, we will couple this approach with the BCGO-method so to recover forces and particles velocities at any-cross-section of a uniform bar without limitation on test duration.

\section{THEORY}

\subsection{Wave propagation}

Firstly, let's consider a uniform viscoelastic bar. We assume that only the fist longitudinal mode was excited. The Fourier components of the displacement at any cross-section are given by [1, 2]:

$$
\tilde{u}(x, \omega)=F(\omega) e^{-I \xi(\omega) x}+D(\omega) e^{I \xi(\omega) x},
$$

where $I$ is such as $I^{2}=-1, x$ is the abscissa of the cross-section, $\omega$ is the angular frequency, $\xi(\omega)$ is the dispersion relation of the bar and $F(\omega)$ and $D(\omega)$ are the Fourier transforms of the forward and 
downward waves at the origin. The Fourier components of the axial particle velocity and the normal force are then obtained as follows:

$$
\begin{gathered}
\widetilde{V}(x, \omega)=I \omega\left(F(\omega) e^{-I \xi(\omega) x}+D(\omega) e^{I \xi(\omega) x}\right), \\
\widetilde{N}(x, \omega)=I S \xi(\omega) E(\omega)\left(-F(\omega) e^{-I \xi(\omega) x}+D(\omega) e^{I \xi(\omega) x}\right),
\end{gathered}
$$

where $S$ is the cross-section area of the bar and $E(\omega)$ is the complex Young's modulus modelling the viscoelastic behaviour of the material. Let's consider these equations at the origin; we can recover the forward and the downward waves:

$$
\begin{aligned}
& F(\omega)=\frac{1}{2}\left(\frac{\widetilde{V}(0, \omega)}{I \omega}-\frac{\tilde{N}(0, \omega)}{I S \xi(\omega) E(\omega)}\right), \\
& D(\omega)=\frac{1}{2}\left(\frac{\widetilde{V}(0, \omega)}{I \omega}+\frac{\widetilde{N}(0, \omega)}{I S \xi(\omega) E(\omega)}\right) .
\end{aligned}
$$

Now, we can substitute the forward and the downward waves in eqs. (2) by their expression given in eqs. (3). We get the following equation which relates the velocity and the force at any cross-section to the force and the velocity at the origin:

$$
\left[\begin{array}{l}
\widetilde{N}(x, \omega) \\
\widetilde{V}(x, \omega)
\end{array}\right]=\left[\begin{array}{cc}
\cos (\xi(\omega) x) & I Z(\omega) \sin (\xi(\omega) x) \\
I \sin (\xi(\omega) x) / Z(\omega) & \cos (\xi(\omega) x)
\end{array}\right]\left[\begin{array}{c}
\widetilde{N}(0, \omega) \\
\widetilde{V}(0, \omega)
\end{array}\right],
$$

where $Z(\omega)=S \xi(\omega) E(\omega) / \omega$ is the complex impedance of the bar. Eqn. (4) which is presented yet in $[1,4]$ is very interesting. Knowing the particle velocity and the force at the origin one can recover these two parameters at any cross-section. Chiefly, the particle velocity and the force at the end of the bar are given by:

$$
\left[\begin{array}{l}
\widetilde{N}(L, \omega) \\
\widetilde{V}(L, \omega)
\end{array}\right]=\left[\begin{array}{cc}
\cos (\xi(\omega) L) & I Z(\omega) \sin (\xi(\omega) L) \\
I \sin (\xi(\omega) L) / Z(\omega) & \cos (\xi(\omega) L)
\end{array}\right]\left[\begin{array}{c}
\widetilde{N}(0, \omega) \\
\widetilde{V}(0, \omega)
\end{array}\right]=[P]\left[\begin{array}{c}
\widetilde{N}(0, \omega) \\
\widetilde{V}(0, \omega)
\end{array}\right],
$$

where $L$ is the bar length. Following Bacon [1], the matrix [P] will be called the transfer matrix of the bar.

Let's consider now a stepped bar made of $n$ uniform segments as showed in figure 1 . The origin of the bar is taken at its left side. Assuming that the particle velocity and the force are continuous along the bar, we get the following relation:

$$
\left[\begin{array}{l}
\widetilde{N}\left(x_{n}=L, \omega\right) \\
\widetilde{V}\left(x_{n}=L, \omega\right)
\end{array}\right]=\left[P_{n}\right] \ldots\left[P_{2}\right]\left[P_{1}\right]\left[\begin{array}{l}
\widetilde{N}(0, \omega) \\
\widetilde{V}(0, \omega)
\end{array}\right] .
$$

In eqn. (6), $\left[P_{i}\right]$ denotes the transfer matrix of $\mathrm{i}^{\text {th }}$ segment. Let be a cross-section labelled $j$ which belongs to the segment $i$ and let $y_{j, i}$ the distance between this cross-section and the left end of the

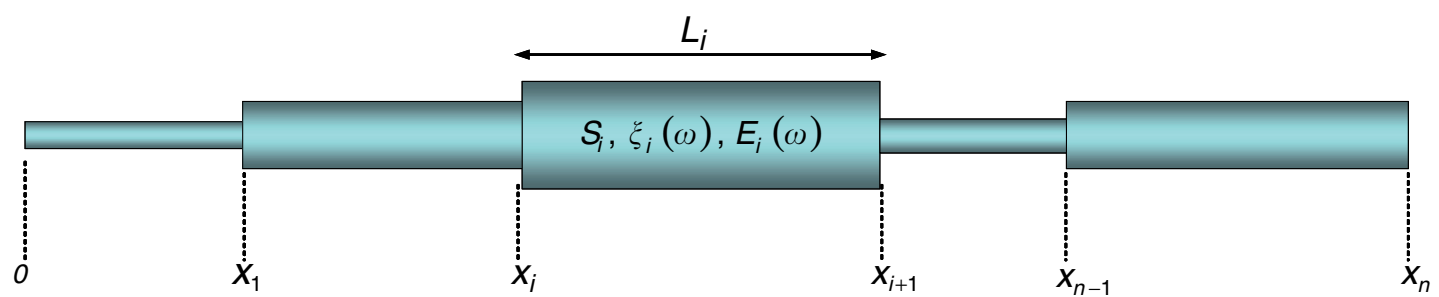

Figure 1. Simplified scheme of a stepped bar. 
segments. The particle velocity and the force are deduced from the particle velocity and the force at the left side of the whole bar as follows:

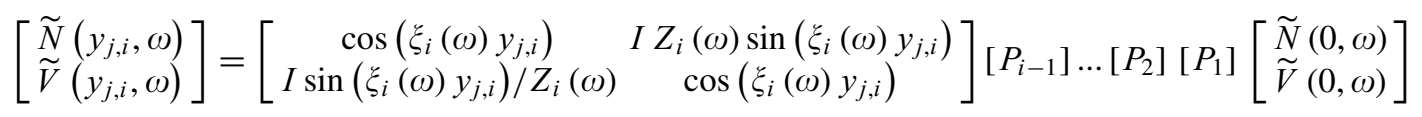

which can also be rewritten:

$$
\left[\begin{array}{l}
\widetilde{N}\left(y_{j, i}, \omega\right) \\
\widetilde{V}\left(y_{j, i}, \omega\right)
\end{array}\right]=\left[\begin{array}{ll}
\alpha_{j}(\omega) & \gamma_{j}(\omega) \\
\delta_{j}(\omega) & \beta_{j}(\omega)
\end{array}\right]\left[\begin{array}{l}
\widetilde{N}(0, \omega) \\
\widetilde{V}(0, \omega)
\end{array}\right]
$$

where $\alpha_{j}(\omega), \beta_{j}(\omega), \gamma_{j}(\omega)$ and $\delta_{j}(\omega)$ are complex frequency-dependent functions.

\subsection{Wave separation}

Multiple strain measurements are supposed to be recorded at $\mathrm{M}$ cross-section $y_{j, i}$. Each measured strain $\hat{\varepsilon}_{j}(t)$ is assumed to be the sum of the actual strain and a white Gaussian noise:

$$
\hat{\varepsilon}_{j}(t)=\varepsilon\left(y_{j, i}, t\right)+w_{j}(t) .
$$

To rebuild the particle velocity and the force at left side of the bar, we will apply the maximum of likelihood method which is based on the assumption that, the observed phenomena (the measured strains) correspond to the most probable event. We consider that noises are two-by-two independents; this method is, in this case, equivalent to the least square method. The solution corresponds to the minimum of the following function [2]:

$$
E=\sum_{j=1}^{M}\left\|\tilde{\hat{\varepsilon}}_{j}(\omega)-\alpha_{j}(\omega) \tilde{N}(0, \omega)-\gamma_{j}(\omega) \widetilde{V}(0, \omega)\right\|^{2}
$$

Therefore the particle velocity and the force satisfy a two-equation linear system:

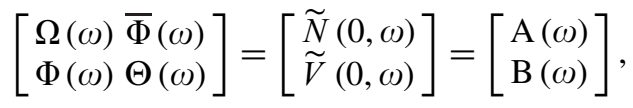

where, $\quad \Omega(\omega)=\sum_{j=1}^{M}\left\|\alpha_{j}(\omega)\right\|^{2}, \quad \Theta(\omega)=\sum_{j=1}^{M}\left\|\gamma_{j}(\omega)\right\|^{2}, \quad \Phi(\omega)=\sum_{j=1}^{M} \alpha_{j}(\omega) \bar{\gamma}_{j}(\omega)$, $A(\omega)=\sum_{j=1}^{M} S_{i} E_{i}(\omega) \bar{\gamma}_{j}(\omega) \tilde{\hat{\varepsilon}}_{j}(\omega)$ and $\mathrm{B}(\omega)=\sum_{j=1}^{M} S_{i} E_{i}(\omega) \bar{\alpha}_{j}(\omega) \tilde{\hat{\varepsilon}}_{j}(\omega)$ and where $\bar{z}$ denotes the complex conjugate of $z$. The solution of the system (11), is:

$$
\begin{aligned}
& \tilde{N}(0, \omega)=\frac{\Theta(\omega) \mathrm{A}(\omega)-\bar{\Phi}(\omega) \mathrm{B}(\omega)}{\Theta(\omega) \Omega(\omega)-\bar{\Phi}(\omega) \Phi(\omega)}, \\
& \widetilde{V}(0, \omega)=\frac{\Theta(\omega) \mathrm{A}(\omega)-\bar{\Phi}(\omega) \mathrm{B}(\omega)}{\Theta(\omega) \Omega(\omega)-\bar{\Phi}(\omega) \Phi(\omega)} .
\end{aligned}
$$

It is worth to notice here that, the denominator of the system (11) may become zero for some frequencies. To avoid this problem, the integration of the Fourier transform will be carried out in the lower half-plane of the complex frequency domain following [2]. 


\section{NUMERICAL VALIDATION}

In this section the new method will be validated using a four-segment elastic bar which is presented in figure 2. The characteristics of each segment are given in the table 1. An impulse load is applied to the left side (the origin) of the bar (figure 3a). The right side is fixed. The impulse duration and the rise-time are taken $46 \mu \mathrm{s}$ and $4 \mu \mathrm{s}$, respectively. Wave propagation solution is computed using an explicit commercial code (Abaqus 6.5-1). The duration of the simulation is 10ms. Looking for clarity, in some figure only the beginning of the simulation is showed. The bar is meshed using one-dimensional linear truss elements. The maximum element length is $1 \mathrm{~mm}$. The maximum time step is $1.07 \mu \mathrm{s}$. It worth noticing, that the computed force, at the left side, is slightly different from the imposed perfect impulse load as shown at the figure (figure 3.b). The strain was recorded at three cross-section of the bar, $\mathrm{y}_{1,1}=300 \mathrm{~mm}, \mathrm{y}_{2,2}=200 \mathrm{~mm}$ and $\mathrm{y}_{3,3}=100 \mathrm{~mm}$, i.e. at the three segment interfaces. The crosssecond is taken to belong to the left segment. The recorded strains are showed in figures 4 .

The force at the left side of the bar is then computed using eqs (13-14). In the figure 5.a, we superpose the force at the origin computed with Abaqus and the force recovered using the wave separation technique at the same cross-section. The slight difference between the two results is due to the numerical

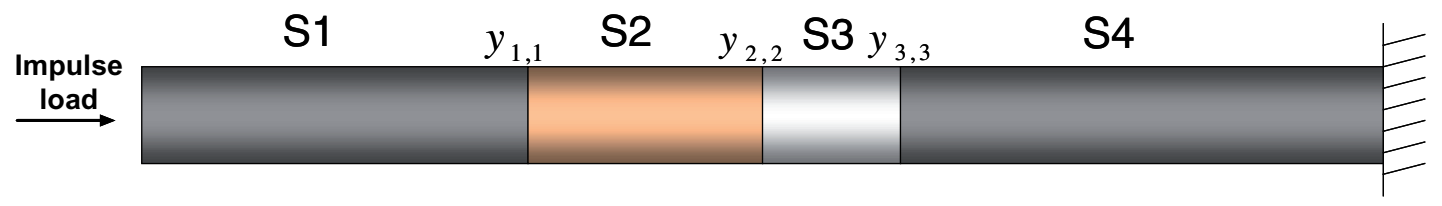

Figure 2. Simplified scheme of the modelled four-segment elastic bar.

Table 1. Characteristics of the modelled four-segment elastic bar.

\begin{tabular}{|l|c|c|c|c|}
\hline Segment & S1 & S2 & S3 & S4 \\
\hline Material & Steel & Aluminium & Titanium & Steel \\
\hline Young's modulus $(\mathrm{GPa})$ & 200 & 70 & 120 & 200 \\
\hline Density & 7800 & 2800 & 4400 & 7800 \\
\hline Cross-section diameter $(\mathrm{mm})$ & 10 & 10 & 10 & 10 \\
\hline Length $(\mathrm{mm})$ & 300 & 200 & 100 & 400 \\
\hline
\end{tabular}

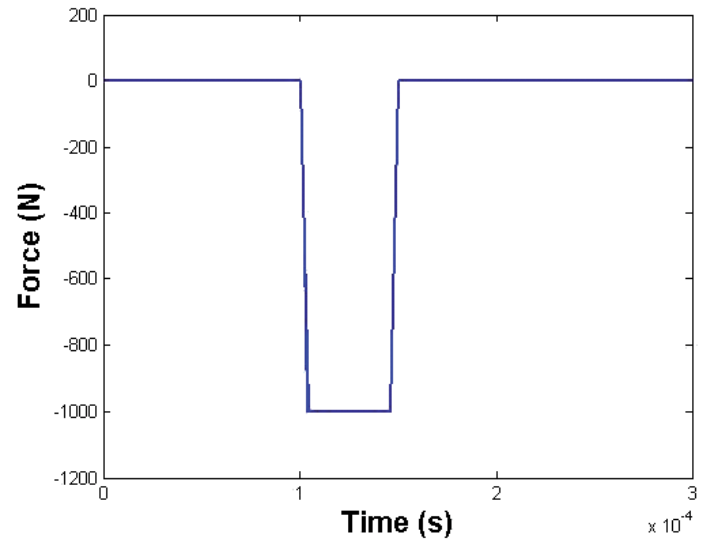

(a)

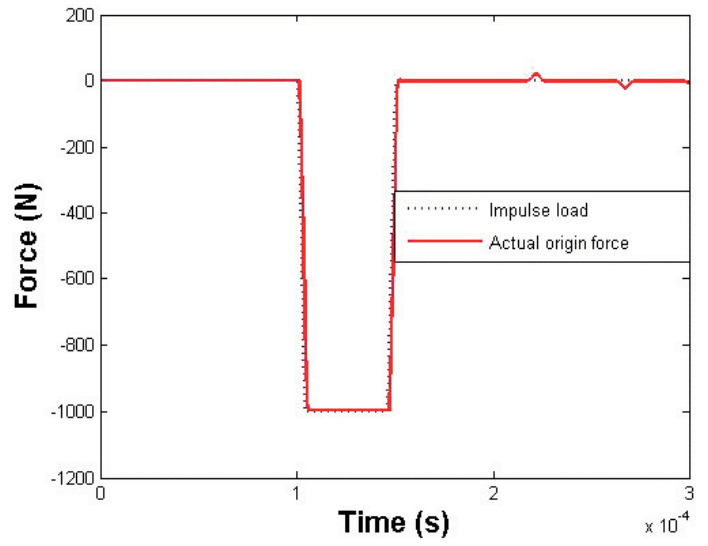

(b)

Figure 3. The force at the bar left side : (a) Imposed impulse load, (b) Force as computed by Abaqus (only the first $300 \mu$ s of the simulation are shown for both figures). 


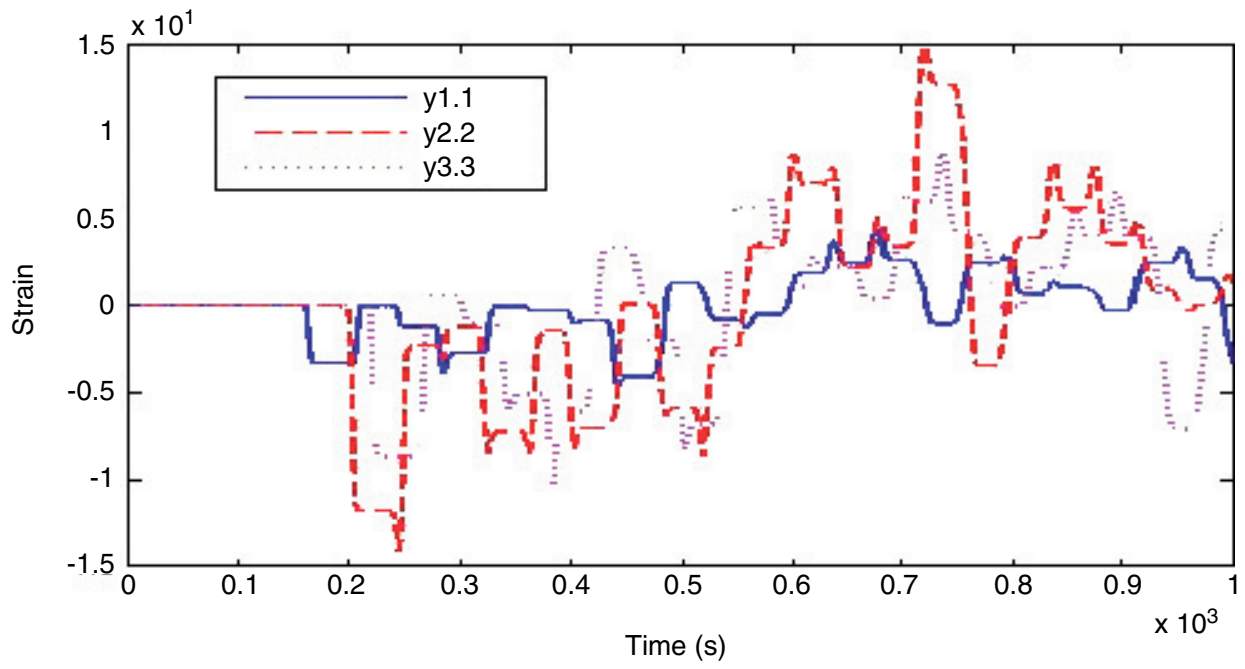

Figure 4. The simulated strains in the bar (only the first $1 \mathrm{~ms}$ is shown).

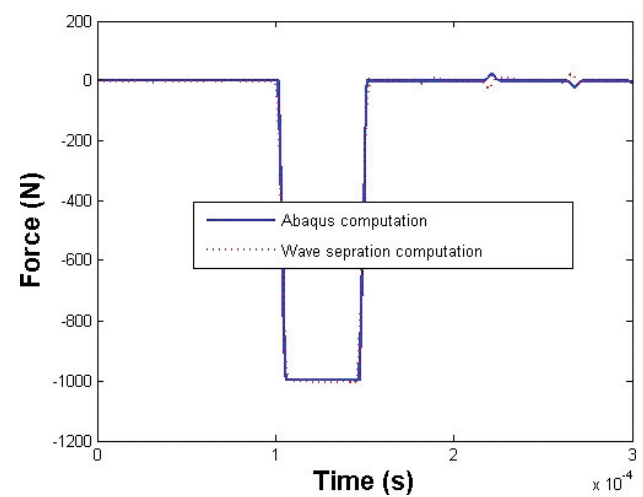

(a)

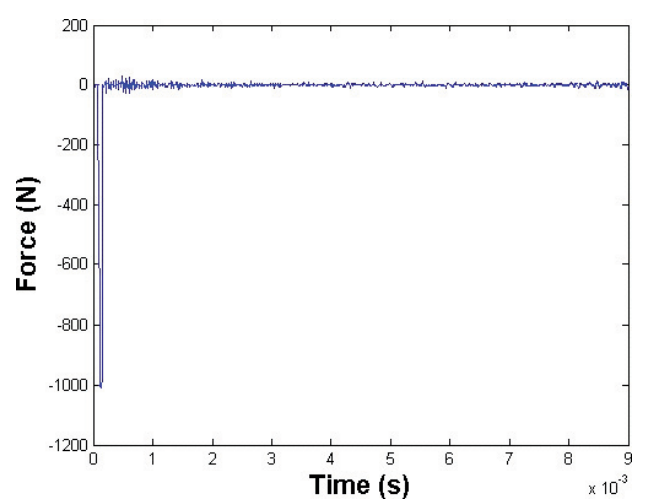

(b)

Figure 5. Recovered force at the origin: (a) the first $300 \mu \mathrm{s}$ (b) the first $9 \mathrm{~ms}$.

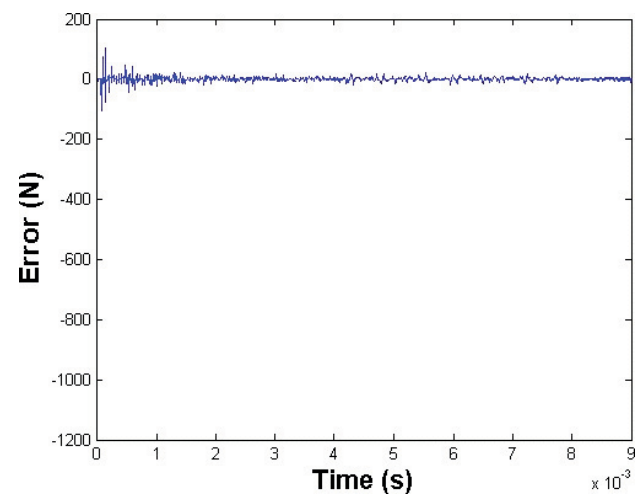

(a)

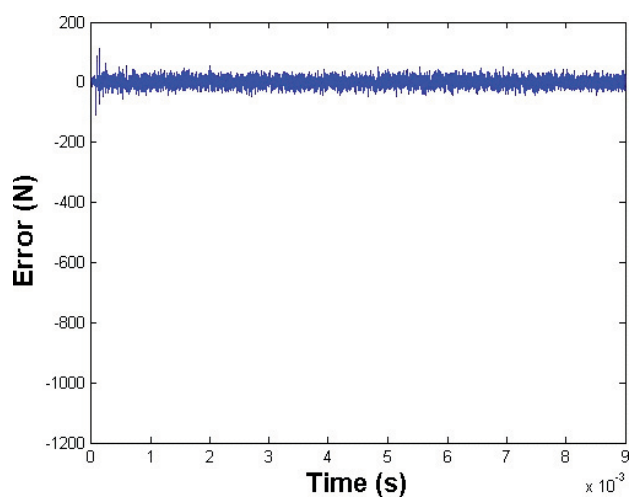

(b)

Figure 6. Error on the recovered force: (a) strains without added noise (b) noised strains. 
errors of the explicit code computation which are not taken into account in the wave propagation model. In figure 5.b, we show the recovered force during $9 \mathrm{~ms}$. The impulse load is well reconstructed. The error (the algebraic difference between the Abaqus signal and the recovered one) is not important (see figure 6.a).

The advantage of the redundant method is its strength against noise. To check this property, a random noise is added to each simulated strain. The noised strains are now used to recover the force at the origin. In Table 2, we present the variance of the error for different values of the variance of the added noise. The wave separation method does not amplify noise much than three times. In figure 6.b, one example is presented. It corresponds to $1.5 \%$-variance added noise.

Table 2. Variation of the error with the added noise.

\begin{tabular}{|l|c|c|c|c|c|}
\hline Variance of the added noise (\%) & 0 & 0.5 & 1.5 & 2.9 & 7.2 \\
\hline Variance of the error (\%) & 0.5 & 1.4 & 3.3 & 6.6 & 17 \\
\hline
\end{tabular}

\section{CONCLUSION}

In this paper we presented an enlargement of the application of the BCGO-wave separation method to non-uniform bars. The technique is valid for elastic and viscoelastic, dispersive and non-dispersive nonuniform bars. It is based on the one-mode wave propagation assumption. Actually, the method is exact for stepped bars. It can be extended to other kinds of non uniformity by sliding the bar in small uniform segments. The method is validated on a numerical model. An experimental validation should follows.

\section{References}

[1] Bacon C. and Brun A., Methodology for a Hopkinson bar test with a non-uniform viscoelastic bar. Int. J. Impact Engng., 24(2000), 219-230.

[2] Bussac M.N., Collet P., Gary G. and Othman R., An optimisation method for separating and rebuilding one-dimensional dispersive waves from multi-point measurements: Application to elastic and viscoelastic bars., J. Mech. Phys. Solids, 50(2002), 321-349.

[3] Jacquelin E. and Hamelin P., Force recovered from three recorded strains., Int. J. Solids Structures, 40(2003), 73-88.

[4] Lundberg B. and Henchoz A., Analysis of elastic waves from two-point strain measurements., Exper. Mech., 17(1977), 213-218.

[5] Lundberg B., Carlsson J. and Sundin K.G., Analysis of elastic waves in non-uniform rods from two-point strain measurement. J. Sound Vibration, 137(1990), 483-493.

[6] Meng H. and Li Q.M., An SHPB set-up with reduced time-shift and pressure bar length. I. J. Impact Engng., 28(2003), 677-696.

[7] Park S.W. and Zhou M., Separation of elastic waves in split Hopkinson bars using one-point strain measurements. Exper. Mech., 39(1999), 287-294.

[8] Zhao H. and Gary G., A new method for the separation of waves: application to the SHPB technique for an unlimited duration measurement. J. Mech. Phys. Solids, 45(1997), 783-493.

[9] Zhao P.J. and Lok T.S., A new method for separating longitudinal waves in a large diameter Hopkinson bar. J. Sound Vibration, 257(2002), 119-130. 